\title{
Why Words Matter: How the Common Mis-use of the Term Music Therapy May Both Hinder and Help Music Therapists
}

\author{
Sarah Pearson ${ }^{1 *}$ \\ 1 Grand River Hospital, Canada \\ *sapearson@gmail.com
}

Received: 2 January 2017 ; Accepted: 1 November 2017 ; Published: 1 March 2018

Editor: Tríona McCaffrey Reviewer: Joanne Loewy

\begin{abstract}
This reflective paper examines occasions where medical studies refer to music therapy as a practice that can be used by non-music therapists. This common use of the term music therapy to describe any use of music for wellbeing, is an area of professional frustration and ethical concern for music therapists. The author explores reasons why the term music therapy is so commonly used to describe something other than the scope of practice; the impact on music therapists of this common misconception are discussed; and opportunities for music therapists to respond positively to these misconceptions are explored.
\end{abstract}

Keywords: music therapy, scope of practice, professional issues, health care professionals, professional boundaries

\section{Introduction}

I recently drafted a literature review for a study, in which nurses were surveyed on their perceptions of the value of music in their care settings. I was surprised and pleased to discover, in preparing this literature review, that there are many published studies on nurses' perceptions of music in care. I was even more surprised to see that, in study after study, music therapy was refered to as a nursing intervention. In these studies, the term music therapy was used to describe using music for wellbeing, by professionals who were not trained music therapists.

"Defining music therapy is an integral part of being a music therapist," wrote Ken Bruscia (1998, p. 1) in his iconic book Defining Music Therapy. Music therapists often find themselves not only being asked to explain what is a very broad and eclectic scope of practice but also having to explain the difference between music therapy and using music for therapeutic or wellness goals.

For the purposes of this paper, I will use the term health musicking hereafter to describe the act of engaging in music for wellness goals. Health musicking is itself a contentious term. Stige (2002) first defined it as a therapeutic intervention:

Music therapy as a discipline is defined as 'the study and learning of the relationship between music and health.' As professional practice it is situated health musicking in a planned process of collaboration between client and therapist. (Stige, 2002, p. 198) 
Bonde (2011, p. 121) proposed that the term could be de-clinicalized, offering one possible definition of health musicking as "the common core of any use of music experiences to regulate emotional or relational states or to promote well-being, be it therapeutic or not, professionally assisted or self-made." Health musicking, he wrote, "cannot be monopolized by 'music therapists', 'community musicians' or 'music and health workers"' (p. 135). A year later, Stige (2012) developed his initial definition of health musicking to now read as "a possible framework for a broader interdisciplinary area of music, health, and wellbeing" (p. 183).

As this brief discussion alone indicates, the challenge of defining terms around music and wellbeing, even umbrella terms, is nothing new.

\section{Defending music therapy: a common fatigue}

As a certified music therapist in Canada, I suffer from the common fatigue shared by many in this profession of constantly re-defining "what is music therapy" for curious colleagues, as well as sometimes correcting the use of the term music therapy when it is used to describe health-musicking activities that aren't delivered by a certified music therapist ${ }^{1}$. It is overwhelmingly common to hear of health-musicking activities described as music therapy in both the media and in colloquial conversation - and it would appear, in peer-reviewed literature as well. For those thousands of clinicians around the world who have dedicated years of expensive training to become music therapists, and who know of the depth that this work can reach, hearing the term music therapy inaccurately used can feel not only devaluing of our scope of practice, but ethically concerning too. The Canadian Association of Music Therapists' (CAMT) Code of Ethics ${ }^{2}$ requires members to accurately represent the profession, and supporting the growth of the profession is something that members are bound to. The American Music Therapy Association (AMTA) requires the same. ${ }^{3}$

In this paper, I wonder: why is the term music therapy so commonly misused? I question: is it necessarily a bad thing? And finally ask: what can music therapists do to respond to this common misunderstanding?

\section{Background}

This reflective paper emerged out of an experience of preparing a literature review of nurses' perceptions of music therapy and music-based interventions. Five of the seven articles I consulted in a brief search used the term music therapy as something that could be delivered either by a nurse, or by a care provider without any specified skillset.

One study (Lok, 2013) from University of Toronto surveyed the perceived value of music among nurses specifically, and concluded with recommendations: "strategies should be implemented by nursing schools and clinical practice areas to ensure that nurses are informed about the effectiveness of music therapy, as well as trained in the application of this intervention in practice" (p. 112). Another study (Sung, Lee, Chang \& Smith, 2011) surveyed nurses' attitudes about music with older adults, concluding: "nursing staff can be the suitable personnel to learn easily and implement music therapy as a part of routine activity programmes for those with dementia" (abstract). A study on gastroenterological nursing concluded that nurses "can offer music therapy" (Chlan, Evans, Greenleaf, \& Walker, 2000, p. 148), and another study on cardiac nursing referred to music therapy as a "nursing intervention" (Taylor-Piliae, Sek-Ying, 2002, p. 203).

Other articles reviewed used the more accurate umbrella term of music medicine, or specified terms such as patient-directed music (Tracy, Chlan, \& Staugaitis, 2015, p. 54), to describe a music intervention delivered by someone other than a music therapist in a therapeutic relationship. In these latter cases, more distinction was made about the difference between music therapy and health musicking. The majority of studies reviewed however used the terms music therapy and music interventions interchangeably, with little detail on the interventions themselves, or who delivered the intervention. 
To make matters more interesting, the benefits of what was called music therapy was backed by extensive literature reviews in many of these articles, with authors citing studies written by certified music therapists. This suggests that these authors a) had access to and awareness of music therapy as a specialized scope of practice, and b) used the term music therapy as an umbrella term. Furthermore, an assumption was made in some articles that music therapy interventions could be administered by nurses, and other practitioners. By contrast, it would be hard to imagine a survey asking a registered dietitian how confident they were to administer a physiotherapy assessment.

It can be cringe-worthy for a music therapist like myself to read study after study referring to music therapy as a generalized use of music for wellbeing, rather than my hard-fought-for profession. And yet, I often walk that fine line between health-musicking and music therapy myself. In addition to my clinical practice as a music therapist in a community hospital and private practice, I train professional and volunteer care providers in the music care approach (Foster, Pearson, Berends, 2016, p. 199). The music care approach is an emerging theory of care practice that supports care providers of all backgrounds to integrate the healing properties of music into their care, in a way that fits with their role and scope of practice. I have developed these courses through my work with a social enterprise dedicated to providing leadership in music care ${ }^{4}$. The courses I offer give baseline training for using the music care approach ${ }^{5}$. Participants spend two days learning the basics of music theory, workshopping singing and humming techniques, going through various musical experiences to internalize of the impact of sound and music on their personhood, and discussing as a group how they could strengthen their current scope of practice with music. Every workshop reviews the Ten Domains of Music Care (ibid) - a framework designed to help locate the multiple uses of music in caregiving - and music therapy is always identified as a scope of practice onto its own.

Many participants come to these workshops expecting to get trained in music therapy and learn quickly that music therapy is something far more specific than they had assumed. Occasionally someone will claim that they are "already doing music therapy" because, for example, they lead a bell choir with residents at a nursing home, and leave the course understanding that their music care work is valuable, though not music therapy. This does not make their work better or worse than music therapy. It simply helps them locate their activity within a taxonomy of music care.

Correcting people's language around music therapy can be tedious, but I keep doing it. I don't like policing people who are doing meaningful work, and I don't like policing music. I would much prefer to define my scope of practice by actually doing the work than by correcting other people for how they define their work. And I would much rather use my role as a music therapist to empower other care providers to embrace the power of music, and elevate the presence of music in all care contexts. Language specificity about roles, however, is important, particularly when we are doing work that has the potential to cause harm (Gardstrom, 2008,, Isenberg, 2012).

\section{Why is the term music therapy so commonly misused?}

Sometimes I casually refer to my sweet 9-year old cat as a "therapy pet" because she is such a sensitive snuggler. While I know that pet therapy is something that people and animals actually train for, the words roll off my tongue. While there is a difference between speaking casually about pet therapy and publishing research studies about it, my casually inaccurate language helps me understand why the term music therapy is so loosely and inaccurately used.

The words music and therapy are not protected terms in Canada where I live, and both are used colloquially. Music points to a broad human phenomenon that is as challenging to define as words like life, death, or beauty. Therapy however, while a clinical term to those of us practicing as therapists, is used commonly in everyday speech in my country as a noun, a verb and an adjective, often used interchangeably with words 
such as wellness or self-care. "Retail therapy","netflix therapy", and "my friend therapized me," are all common examples of this. The term also pops up in retail products such as, for example, "therapeutic bath bombs" and "aromatherapy candles." We are a far cry from being able to regulate the use of the word therapy.

The wellness benefits of music are so inherently understood by many that the term music therapy would roll naturally off tongues, just as pet therapy does mine. Ask a teenager about the music on their device, and you'll often hear them say something akin to "music is my therapy." It makes sense to me and I understand what they mean. Ken Bruscia (ibid) echoed this, saying that "defining therapy is as difficult as defining music." (9)

And then there is the issue of the profession itself. In many parts of the world, music therapists aren't part of a unified scope of practice. In Canada, the Canadian Association of Music Therapists is still in the process of defining a scope of practice. Music therapists work in incredibly diverse settings; our jobs looking significantly different from one to the other, in terms of content, context, and salary. We have sub-specialties like neurologic music therapy (NMT), Nordoff-Robbins, and vocal psychotherapy, and other credentials such as registered psychotherapists. There is grey area in much of what music therapists do. Bruscia (1998) emphasized this, pointing out in the first edition of his seminal book Defining Music Therapy that the difficulties of defining the profession include that music therapy is by nature "transdisciplinary," straddling fields as broadly-ranging as psychotherapy, clinical theory, psychoacoustics, entertainment, medicine, healing traditions, and social work, to name just a few (p. 7). Other issues include that the field is "incredibly diverse," (p.11), that there is a dual identity of "discipline" and "profession," (p.14) and that music therapy "is still developing" as a discipline (p. 15).

\section{Is the confusion of terms a bad thing?}

The lack of clarity about the term music therapy in public perception - as well, as it would appear, in the scientific journal articles I reviewed that used the term music therapy incongruently defined against its own cited literature - raises obvious concerns for certified music therapists. It means potential employers may not understand the parameters of our scope of practice and that the public may not understand how they could benefit by accessing a music therapist. It means our jobs may be harder to maintain and our pay scale harder to standardize. If clinician-scientists are citing music therapy literature and still missing the point that music therapy is a specialized practice, this is a discouraging sign for music therapists trying to trail-blaze in health care settings.

As clinicians, we recognize the potential for our practice to cause harm as well as good (Isenberg, 2012), and this is makes it all the more important that the public know what service it is that they are accessing. If someone is paying for what they have been told is music therapy, then music therapists are ethically invested to ensure that they are actually receiving the accurate treatment.

And yet, inherent in the generalized use of the term music therapy is an implied valuing of music. Returning to the pet-therapy analogy, when I refer to my cat-cuddles as pet therapy I am essentially praising the wellness-factor of pets. By casually attaching the word therapy to music, the value of music for wellbeing is being inferred. Music as a means of care is being elevated. In their inaugural issue, the editors of the International Association for Music and Medicine's $\left(\mathrm{IAMM}^{6}\right.$ ) Music and Medicine Journal identified this elevation of music in health care as a rationale for founding their publication:

In the past decade, we have witnessed a surge in the number of studies that have integrated the science of medicine with the art of music, and the art of medicine with the science of music...With the surge of "integrative" acceptance in medical practice, the expansion of medical music interventions has been much more easily understood ... Music and Medicine is recognizably adherent to the growing realization that interdisciplinarity, and par- 
ticularly the surge of integrative models, is evident in the forefront of modern health care thinking worldwide. (Loewy \& Aldridge, 2014, p. 5-6)

Acknowledging that the acceptance of music within integrated health care is growing does not in-itself help define music therapy specifically. But it does demonstrate an emerging value of music as a wellness tool, something which music therapists can capitalize on.

\section{What can music therapists do?}

Capitalizing on this perceived value of music requires skillsets that may be unfamiliar or uncomfortable to the average clinician. The inherent misunderstanding of the scope of practice by the general public means that music therapists often have to become public relations experts, sales people, and entrepreneurs, as well as skilled therapists. This is unrealistic and often ethically complicated as conflicts of interest can emerge when we have to wear both business and clinical hats.

However, I propose that as music therapists, we have an opportunity to position ourselves as experts to care communities that are already excited about music. Perhaps our professional role can become two-fold: we can be music therapists, as well as advocates for music as a wellness tool. Opportunities for inter-professional collaboration is ripe, and this may require us to embrace the dual-role of clinician and music-expert.

Music therapists can respond to these misunderstandings by taking a leadership role with other care partners who are excited about the potential for music, albeit misinformed about the profession of music therapy. Capitalizing on the interest and enthusiasm for health musicking, music therapists can become the music care "experts" in their communities and build relationships with other professionals through this shared interest in music for wellbeing. Music therapists can offer trainings, in-services, and education to care partners on the role of music and in so doing, create opportunities to demonstrate specific examples of music therapy, helping to educate and elevate the profession, while also empowering other care partners with music.

The IAMM has taken this very approach, by opening a scholarly discussion that embraces the interdisciplinary interest in music and health. By sitting on the fault-line of ambiguity between music and health care, clearer definitions of differing music interventions emerge, and relationships between music-based practitioners and medicine grow stronger. With an interdisciplinary editorial board that has a heavy presence of music therapists, the IAMM sets a promising tone for embracing expertly-driven interdisciplinarity:

We are seeing increasing specialties of particularized knowledge. These areas of interest include but are not limited to arts medicine, music performance, performance arts medicine, music psychology, ethnomusicology, music cognition, music neurology, music therapy, music in hospitals, early childhood and developmental music education, infant stimulation, and music medicine. Our initiative in Music and Medicine will be to promote translational research - that is, to promote understanding that will further the field of music and medicine. (Loewy \& Aldridge, 2014, p. 7)

Another approach that music therapists can take is to let go of monitoring the term music therapy and instead promote and strengthen the term music therapist. The CAMT is moving in this direction, having passed a motion in 2016 to change the name of the organization from Canadian Association of Music Therapy to Canadian Association of $\mathrm{Mu}$ sic Therapists. This change reflects a re-prioritizing of the organization's role - to focus more attention on protecting and promoting the term music therapists rather than the term music therapy.

\section{The Current Climate}

The issue of language is timely. Attempts are being made to make language about health musicking clearer. The IAMM is just one example of attempts to grow clearer about music interventions through highlighting the diverse ways that music can be 
integrated into care. It is a beginning of branching away from mis-naming all music interventions music therapy. Bonde (2011) summarized the challenge succinctly:

I think we need some orientation tools, as the field of music, culture and health is rapidly growing and becoming potentially confusing. Music therapists have fine qualifications to work in the field, ... but the field ... is notoriously open to anyone who wants to contribute to musicing for health purposes. (p. 133)

A 2012 book by McDonald, Kreutz and Mitchell compiled multidisciplinary articles "echoing the huge interest in the relationships between music, health and wellbeing" (p. 7). Recognizing the need to be able to categorize different types of health-musicking activities, the authors propose a conceptual framework they define as "a multitude of [health-musicking] approaches and many different epistemologies" (p. 8). The framework proposes four overlapping categories, including music education, music therapy, community music, and everyday uses of music. This need for a framework was reflected in my own co-authored paper on the Ten Domains of Music Care (Foster et al., 2016), which presents 10 categories for mapping health musicking activities, including a stand-alone category for music therapy.

\section{Conclusion}

The generalized use of the term music therapy may indeed be a problem that is embedded in language itself and something inherent to the profession. We can be hopeful that over time the profession will become more recognized and the term consequently more protected. Looking for the opportunities for advancing not just the profession of music therapy, but the broader use of music in care, can be a positive outcome of what has been a chronic issue of concern for the music therapy profession. The fear of losing the expert-specificity of music therapy can be one impediment to music therapists' embracing a leadership role in the use of music across disciplines. Yet taking this interdisciplinary leadership role as health-musicking experts is one way of raising awareness of the profession. And remembering that each inaccurate use of the term music therapy is also, usually, a great compliment to the power of music, and a sign that the culture of care is well on its way to fully embracing the valuable service we offer.

\section{Notes}

1. In Canada, a minimum of 2 years' post-secondary education, in addition to prior musical training, plus a 1000-hour clinical internship, are required for accreditation by the Canadian Association of Music Therapists.

2. Visit http://www.musictherapy.ca for more information.

3. Visit http://www.musictherapy.org for more information.

4. See http://www.room217.ca for more information about this organization.

5. See http://www.room217.ca/music-care-certificate-program for more information.

6. http://www.iammonline.com/

\section{References}

Bruscia, K. (1998). Defining music therapy. Gilsum, NH: Barcelona Publishers.

Bonde, L. O. (2011). Health musicing: Music therapy or music and health? A model, empirical examples, and personal reflections. Music and Arts in Action, 3(2), 120-140.

Chlan, L., Evans, D., Greenleaf, M., \& Walker, J. (2000). Effects of a single music therapy intervention on anxiety, discomfort, satisfaction, and compliance with screening guidelines in outpatients undergoing flexible sigmoidoscopy. Gastroenterology nursing: the official journal of the Society of Gastroenterology Nurses and Associates, 23(4), 148-156, https://doi.org/10.1097/00001610-200007000-00003. 
Foster, B., Pearson, S., \& Berends, A. (2016). Ten domains of music care: A framework for delivering music in Canadian healthcare settings. Music and Medicine, 8(4), 199-206.

Gagner-Tjellesen, D., Yurkovich, E., \& Gragert, M. (2001). Use of music therapy and other ITNIs in acute care. Journal of Psychosocial Nursing and Mental Health services, 39(10), 26-37.

Gardstrom, S. C. (2008). Music therapy as noninvasive treatment: Who says? Nordic Journal of Music Therapy, 17(2), 142-154.

Isenberg, C. (2012). Primum nil nocere (Above all, do no harm): A direction for the development of music therapy. Canadian Journal of Music Therapy, 18(1), 62-78.

Lok, J. (2013). Nurses awareness of and intention to use music therapy in practice. University of Toronto. Unpublished Master's Thesis.

Loewy, J., \& Aldridge, D. (2014). Prelude to music and medicine. Music and Medicine, 1(1), 5-8, https://doi.org/10.1177/1943862109338696.

MacDonald, R., Kreutz, G., \& Mitchell, L. (2012). Music, health \& wellbeing. London, England: Oxford University Press. https://doi.org/10.1093/acprof:oso/9780199586974.001.0001.

Stige, B. (2002). Culture-centered music therapy. Gilsum, NH: Barcelona.

Stige, B. (2012). Health musicking: A perspective on music and health as action and performance. In R. MacDonald, G. Kreutz, \& L. Mitchell (Eds.), Music, health and wellbeing (pp. 183-195). London, England: Oxford University Press. https://doi.org/10.1093/ acprof:oso/9780199586974.003.0014.

Sung, H. C., Lee, W. L., Chang, S. M., \& Smith, G. D. (2011). Exploring nursing staff's attitudes and use of music for older people with dementia in long-term care facilities. Journal of Clinical Nursing, 20(11-12), 1776-1783, https://doi.org/10.1111/ j.1365-2702.2010.03633.x.

Taylor-Piliae, R. E., \& Chair, S-Y. (2002). The effect of nursing interventions utilizing music therapy or sensory information on Chinese patients' anxiety prior to cardiac catheterization: A pilot study. European Journal of Cardiovascular Nursing, 1(3), 203-211, https://doi.org/ 10.1016/S1474-5151(02)00037-3.

Tracy, M. F., Chlan, L., \& Staugaitis, M. A. (2015). Perceptions of patients and families who received a music intervention during mechanical ventilation. Music and medicine, 7(3), 54-58. 